\title{
PEDAGOGICAL DESIGN ASPECTS OF THE RESPIRATORY DISTURBANCES PROPHYLAXIS IN THE CHILDHOOD
}

\author{
G. Terzieva* \\ Faculty of Education, Trakia University, Stara Zagora, Bulgaria
}

\begin{abstract}
One of the serious problems of the contemporaneous life is the non-complete and non-rational breathing in the childhood. Furthermore, the continuous harmful impact of the environment factors and the way of life loads additionally the children's respiratory system. A negative tendency toward an increasing spread of respiratory diseases in the childhood is observed

The purpose of the present study is to describe a methodology for the pedagogy students training for work with children and pupils suffering from different disturbances of the respiratory function.

Hereinafter we make an attempt at systemizing the breathing exercises according to their effect and at finding the possibilities of their application in the different forms of the motor education.
\end{abstract}

Key words: rational breathing, breathing exercises, pedagogy students

\section{INTRODUCTION}

The main function of the respiratory system is to make a gas exchange in the body providing it with the necessary oxygen and releasing the useless carbon dioxide. All this is made as a result of the close interaction with the organs of the cardio-vascular system (1).

Lungs have not their own musculature. They follow the movements of the thorax and the diaphragm.

The inspiration is an active act, i.e. when contracting the intercostals muscles the thorax expands in a front-rear and lateral direction and when contracting the diaphragm, the vaults of which descend, the vertical size of the thoracic cavity increases and at the same time the abdominal wall is inflated forward. The pressure changes and the lungs are inflated with air, too. The expiration is mainly passive and it depends on the tissues elasticity being helped by the gravitation, the body position and the auxiliary respiratory musculature (2).

There are three types of breathing depending on the degree of participation of the different muscles in the respiratory process.

*Correspondence to: Galena Terzieva,, Faculty of Education, Trakia University - Stara

Zagora,Bulgaria,gtterzieva@abv.bg
1. Abdominal /diaphragm/ breathing - mainly the diaphragm takes part in it and the lungs bottom is inflated.

2. Thoracic /costal/ breathing - It is made by contracting the intercostals musculature. The air penetration is less than during the abdominal breathing and mainly the medium and the top part of the lungs is inflated.

3. Complete /mixed/ breathing - the thorax and the diaphragm participate simultaneously or consecutively $(2,3)$.

The breathing which is considered to be the most effective and correct is the rhythmic, uniform, tranquil and complete breathing in which all parts of the respiratory system participate (3).

Children breathe not economically and superficially. From the age of 3 to 7 years the thoracic breathing dominates and from 8 to 10 years the sex differences begin to manifest themselves - boys breathe mainly by the diaphragm and girls - by the thorax. Their respiratory system does not have enough reserves, the mobility of the thorax is insufficient and the elastic forces are insignificant (4).

The studies of the respiratory differences /an indication of physical development/ made during the last years show that children use almost the lungs entire capacity when in repose. An 
additional physical loading will quickly lead to a fatigue and even may be dangerous for the children's health because their respiratory system will not be able to respond adequately to the increased requirements. One of the main causes for that is the hypodynamia. Furthermore, children of today live in an ecologically polluted environment with a decreased oxygen content in the air which additionally makes difficult the most important vital function for life and health - the breathing (5).

The non correct, non effective and non rational breathing has an unfavorable influence not only on the respiratory apparatus but on the human organism as a whole. The non correct breathing leads to an insufficient ventilation, weakens the respiratory system stability and increases the predisposition to lung diseases which are the most spread diseases in the childhood (3). Most often are the inflammations of the respiratory tract or of the lungs which may become complicated and chronic - chronic bronchitis and chronic pneumonia. Another chronic disease with a social significance disturbing the lungs function is the bronchial asthma.

The motor education in the kindergartens and at the initial stage of the primary education is carried out by the kindergarten and primary teachers. The breathing exercises are an integral part of it. But do children breathe completely and correctly during their motor activity? Are the breathing exercises prophylaxis functions used?

We think that it is possible to answer these questions by optimizing the education of the pedagogy students including a Breathing Module in the facultative subject Motor Training and Kinesitherapy which is a part of the scholar plan of the specialties Preschool and Primary School Pedagogy and Primary School Pedagogy with Foreign Language.

As a result of the education it is expected the following: the students should extend their knowledge about the breathing and the opportunities given by the breathing exercises; they should acquire skills in teaching children to breathe rationally and correctly; they should make a respiratory disturbances prophylaxis.

The correct breathing (3) mastering is submitted to a motor habit creation. It is a respiratory motor habit which is more complex because besides the correct breathing habit the breathing
- motion habit is created. When creating it the following sequence should be observed:

1. Correct breathing mastering in repose.

2. Combination of the breathing and automated movements such as walking and running together with exercises which are simpler and require less efforts during their execution.

3 . Inclusion of more complex exercises and a physical loading increase.

In terms of practice Karanashev (6) generally divides the breathing exercises according to a couple of indications:

1. According to their effect they are as follows:

- with a general effect /with a general influence/

- they have a bigger influence on other organs and systems such as the cardio-vascular and the nervous system. They are the following: breathing exercises with the participation of the upper limbs; of the lower limbs; of the upper and lower limbs; of the upper and lower limbs and the torso; home and professionally applicable breathing exercises the purpose of which is to make automotive the dynamic stereotype of breathing - movements.

- specific - influencing purposefully a defined function or a part of the respiratory apparatus. Besides their general influence they may have more significant therapeutic effect - influencing the rhythm, assisting the expectoration etc.

2. According to that whether they are made only with the participation of the main respiratory muscles or in combination with physical exercises for the different parts of the body they are as follows:

- static /main/ - they are executed only with the participation of the thorax and the diaphragm; according to the breathing type they are exercises for thoracic, for diaphragm and for complete breathing;

- dynamic /combined/ - the breathing is combined with the body movements. They are as follows:

- according to the initial position - from an occipital lying position, side lying position, kneeling position, sitting position, standing position etc.;

- according to the participation of the different body parts and muscular groups - upper limbs, lower limbs, torso or a combination of them;

- according to the activity type - a combination of the breathing and the everyday life activities or the professional activity;

- according to the intensity - maximal, submaximal, moderate, low etc.;

- according to the apparatuses use - sticks, balls, dumb-bells etc. 
3. According to that whether the breathing is directed from outside or not:

- calm, free breathing - i.e. the usual for human beings;

- directed breathing or conscious, intentional, directed breathing - the breathing is slower, deeper, with pauses etc. according to the given instructions. .

4. According to that whether a full ventilation of the lungs is executed or mainly of some parts of them:

- complete breathing;

- breathing with a ventilation of different parts of the lungs /upper thoracic, dorsal, flank etc/.

The respiratory disturbances are related mainly to the respiratory act modification. They are ventilation disturbances and alveolar respiratory disturbances. Ventilation is the most important function which is directly accessible and susceptible to compensatory mechanisms. The ventilation disturbances are divided as follows: obstructive - their symptoms are the clearing decrease and the disturbance of the respiratory tract passability, the increased secretion with an impossibility of expectorating /bronchitis, bronchial asthma etc./; restrictive - a part of the respiratory surface is damaged and it is excluded from activity /pneumonia, tuberculosis, abscess etc/; mixed (3).

The alveolar - respiratory disturbances are the following: a disturbance of the air distribution in the lungs - emphysema; a connective tissue increase in the lungs and a diffusion disturbance - silicosis, fibrosis (3).

The correct breathing is the best prophylaxis of many diseases. Furthermore it may have a multiple curing effect. As a result of the respiratory system diseases and some other diseases the respiratory function may become weakened or disturbed (7). The physical exercises are one of the means that may physiologically strengthen the respiratory system, stimulate and normalize its function and have a curing and prophylaxis effect. When they influence directly the respiratory organs they are called respiratory exercises (6).

According Petkov (2) the specific therapeutic respiratory exercises are the following:

1. Exercises for a nose breathing improvement /slow nose inspiration and expiration, nose inspiration and mouth expiration, mouth inspiration and nose expiration, inspiration through the one nostril and expiration through the other, inspiration with nostrils tapping, breathing with narrowed nostrils, sniffing breathing etc./ and for a respiratory tract passability improvement /for nose breathing improvement, for expectoration and coughing improvement; when there is a respiratory tract spasm - relaxation exercises, sound exercises pronunciation of ,ж”, ,, 3 " etc./.

2. Exercises for an expiration improvement and a weakened breathing stimulation.

The purpose is to activate mainly the expiration, because the maximal expiration will lead by reflex to a more active and deeper breathing. Exercises with the expiration of different consonants are used /,,x”, „ш”, „ф”, ,3”, ,ח”/ and vocals /,„a", ,e”, ,и”, ,o”, ,y"/.

3. Exercises for a respiratory rhythm restoration and regulation. The purpose is to create habits for a correct and continuous breathing with an optimal rhythm. The following exercises are used: inspiration and expiration with counting, inspiration and expiration with a words or sounds pronunciation, inspiration and expiration with walking /inspiration and expiration at a determined number of steps/, inspiration and expiration combined with rhythmic exercises, slow inspiration /at four times/ and quick expiration, quick inspiration and slow expiration, inspiration by pushes and smooth expiration, smooth inspiration and expiration by pushes etc.

4. For the respiratory musculature training exercises with a resistance during the inspiration /the thorax is pressed by hands/ and the expiration /balloon blowing, expiration through a small tube, expiration in water, expiration by pushes etc./ are used; for the thorax mobility improvement respiratory exercises from different initial positions are used - standing position, sitting position; for a vertebral column flexibility increase in all planes influencing directly the thorax mobility and improving the respiratory movements; for the respiratory musculature strengthening exercises with apparatuses are used - a dense ball, small dumb-bells or a gymnastic wall.

5. Exercises for the diaphragm mobility improvement and strengthening. Different initial positions are used - from an occipital lying position with folded legs, in a side lying position, from a lying position and a kneeling position. The diaphragm side on which one is lying is more movable. All exercises using a resistance against the expired flow train the diaphragm. When the diaphragm breathing has been mastered very well exercises for complete breathing may be applied, too.

6. In order to restore or improve the disturbed lung ventilation some special respiratory 
exercises with directed breathing are mainly applied. They have a determined sequence. They begin with the expiration final stages mastering. After then respiratory exercises with an obligatory deepness and frequency apply. Later gymnastic exercises are added during which the breathing is more frequent and deeper. After the state amelioration walking and even slow running with a determined breathing rhythm combined with the tempo may apply. Exercises with a change of the breathing intensity and rhythm are also suitable.

7. In order to suppress the cough an air retaining for a short time and a careful expiration are used. In order to help the expectoration an initial sitting position slightly inclined forward is used executing a deep breathing. The sound respiratory exercises with an expiration of ,,3", „p", the exercises creating a resistance to the inspiration /nostrils narrowing, smelling exercises, sniffing exercises/ and the exercises creating a resistance to the expiration /expiration of „c”, „ф”, candle blowing, balloon blowing, blowing through a tube in a vessel with water/ stimulate the expectoration.

If the respiratory diseases are heavier kinesitherapeutic procedures are administrated. But their treatment may continue at school during the therapeutic physical exercises classes, a weekly form of a motor education using purposefully the breathing exercises therapeutic functions.

The respiratory exercises are an integral part of the main forms of the motor education - the physical education occupation and lesson. They are used mainly for the load regulation and the therapeutic tasks solving - the thorax mobility increase and the respiratory muscles strengthening; the optimization of the state and the correlation of the processes of retention and stimulation of the nervous system assisting its functional disturbances treatment; the increase of the immunologic reactivity of the organism against viruses and bacterial infections; the normalization of the other organs and systems function. All these things may be carried out only if the children breathe correctly and completely, i.e. the respiratory motor habit formation may be included in the motor training as an educational task.
In order to use more completely the prophylaxis functions of the breathing exercises the everyday forms of the motor training may be used - the morning gymnastic and the physical exercises minute and break.

The physical exercises included in the morning gymnastic are usually very well known by the children. If one pays attention to the breathing during their execution, i.e. to combine them with the movements they will become dynamic breathing exercises. When executing them in the open air the influence on the respiratory function is much better.

The recreational character of the physical exercises minute and break may increase when combining the exercises with the breathing.

By enlarging their competences in the field of breathing the pedagogy students will be prepared for realizing the respiratory disturbances prophylaxis in the childhood during their future work as teachers.

\section{REFERENCES}

1. Piryova, B., Nachev, N. Human physiology, Publishing House Arso, Sofia, 2006

2. Petkov, I. Principles of the kinesitherapy Part two, Publishing House Tip-top pres, Sofia, 2008; 94-108

3. Karaneshev, G. Theory and methodology of the therapeutic physical exercises, Publishing House Medicine and Sports, Sofia, 1991; 52-58

4. Boeva, B., Ivanova, V., Theory and methodology of the physical education. Manual for the students from Trakia University. Theoretical and methodological principles of the physical education. Part one. Stara Zagora, 2012; 237-238

5. Popivanova, C., Socially significant diseases and adaptation capacities of the 6-18 years old pupils from Sofia, Sofia, 1995.

6. Karaneshev, G., Practical manual of therapeutic physical exercises. In Bulgarian), Publishing House Medicine and Sports, Sofia, 1991; 30-47

7. Shulpina, V. P., Breathing gymnastic in the process of the therapeutic physical training of pupils, Publishing House SibGUFK, Omsk, 2007; 4 